Pacific Journal of Mathematics

TIE ON N AN EXTREM ForM 


\section{NOTE ON AN EXTREME FORM}

\section{Manoranjan Prasad}

The purpose of this paper is to find a positive definite quadratic form $f_{n}\left(x_{1}, x_{2}, \cdots x_{n}\right)$ which is extreme and for which each of the binary form $f_{2}\left(x_{i}, x_{j}\right)$ is an extreme form. In other words we intend to seek an extreme $n$-ary form $f_{n}\left(x_{1}, x_{2}, \cdots x_{n}\right)$ which remains extreme when it is reduced to a binary form $f_{2}\left(x_{i}, x_{j}\right)$, by setting all but two of the $x$ 's equal to zero.

Let $f_{n}\left(x_{1}, x_{2}, \cdots x_{n}\right)$ be a quadratic form in $n$ variables,

$$
x_{1}, x_{2}, x_{3} \cdots x_{n} \quad: f_{n}\left(x_{1}, x_{2}, \cdots x_{n}\right)=\sum a_{i j} x_{i} x_{j}
$$

with determinant $D=\left|a_{i j}\right|$ and $a_{i j}=a_{j i} \cdot f_{n}\left(x_{1}, x_{2} \cdots x_{n}\right)$ is positivedefinite that is the roots of the characteristic equation

$$
\left|a_{i j}-\lambda \delta_{i j}\right|=0
$$

are all positive, where

$$
\delta_{i j}=1 \quad \text { if } i=j ; \delta_{i j}=0 \text { if } i \neq j .
$$

Let $M$ denote the minimum value of $f_{n}\left(x_{1}, x_{2} \cdots x_{n}\right)$ for integers $x_{1}, x_{2}, \cdots x_{n}$, not all zero. This $M$ is the same for all forms derived from $f_{n}\left(x_{1}, x_{2} \cdots x_{n}\right)$ by unimodular linear transformations. Let $2 s$ denote the number of times this minimum is attained that is the number of solutions of the Diophantine equation:

$$
f_{n}\left(x_{1}, x_{2}, \cdots, x_{n}\right)=M
$$

Let $2 s$ sets of (1.3) be given by

$$
X= \pm M_{k}= \pm\left(m_{1 k}, m_{2 k}, \cdots, m_{n k}\right)
$$

(known as minimal vectors) where $k=1,2, \cdots, s$.

Taking one of the two sets, considered not distinct, we have

$$
\begin{aligned}
\sum a_{i j} m_{i k} m_{j k} & =M \\
k & =1,2, \cdots, s .
\end{aligned}
$$

We consider (1.5) as equations in $a_{i j}$ and suppose that (1.5) has an infinitude of sets of solutions in $a_{i j}$. This means that the auxiliary equation

$$
\sum p_{i j} x_{i} x_{j}=0
$$

with $p_{i j}=p_{j i}$, has an infinitude of sets of solutions. 
Let us define $g(X)=\sum p_{i j} x_{i} x_{j}$ and write $h(X)$ as

$$
h(X)=f(X)+p g(X)
$$

$h(X)$ is positive-definite if $p$ lies in a certain interval $-\delta^{\prime}<p<\delta$. If $\delta=+\infty$ we then find that $-\delta^{\prime}$ is finite and then changing $g(X)$ to $g(-X)$ we get the interval $-\delta<p<\delta^{\prime}$. G. Voronoi has shown that the set $h(X)$ with $0<p<\delta$ contains a form

$$
f^{\prime}(X)=f(X)+P_{1} g(X)
$$

such that the minimum of $f^{2}(X)$ is also $M$ and has all the representations of $f(X)$ and at least one more representation. Hence there is a series $f, f^{1}, f^{2}, f^{3}, \cdots$ of positive definite quadratic forms such that if $S_{r}$ is the number of representations of the minimum $M$ of $f^{r}$ then

$$
S<S_{1}<S_{2} \cdots<S_{r}<S_{r+1} \cdots .
$$

It is known that the number of representations of the minimum of an $n$-ary positive definite quadratic form is at most $2^{n}-1$. Hence the series (1.9) terminates say with $f^{r}$, then $f^{r}$ is determined by its minimum and the representations of its minimum. It is obvious that (1.5) has a unique solution if

$$
S \geqq \frac{1}{2} n(n+1)
$$

We call $f^{r}$ a perfect form.

A perfect form $f(X)$ is said to be extreme if and only if it is eutactic, i.e; if its adjoint $F(X)$ is expressible as

$$
F(X)=\sum_{1}^{s} o_{k} \sum\left(m_{i k} y_{i}\right)^{2}
$$

where all the $\rho_{k}$ are positive.

2. We may replace $f_{2}$, if need be, by an equivalent $f_{2}$ (by applying an unimodular integer substitution) to secure that $f_{2}\left(x_{i}, x_{j}\right)$ is reduced. Combined with $x_{r} \rightarrow x_{r}(r \neq i$ or $j)$, the substitution is unimodular and integer in the full $n$ variables and therefore converts $f_{n}$ to an equivalent $f_{n}$. Since extreme forms remain extreme under the group of unimodular integer transformations, $f_{n}$ and $f_{2}$ still remain extreme. Finally we may take

$$
f_{2}\left(x_{i}, x_{j}\right)=a_{i i} x_{i}^{2}+2 a_{i j} x_{i} x_{j}+a_{j j} x_{j}^{2}
$$

which is reduced and extreme for every $i \neq j$.

3. By the principle of homogeneity, we may take, without loss of any generality the minimum $M$ of $f_{n}\left(x_{1}, x_{2} \cdots x_{n}\right)$ as unity. 
It is well-known that all binary extreme forms constitute a single class of forms equivalent to $x^{2}+x y+y^{2}$. Two positive definite binary quadratic forms $f$ and $f^{\prime}$ of the same determinant are equivalent if and only if their respective reduced forms $\phi$ and $\phi^{\prime}$ are either identical or form one of the special pairs of equivalent reduced forms. Therefore the form (2.1) and $x^{2}+x y+y^{2}$ are of the same determinant and (2.1) is equivalent to $x^{2}+x y+y^{2}$ if and only if the form (2.1) is identical with either of $x^{2} \pm x y+y^{2}$. Alternatively, as $a_{i i} x_{i}^{2}+2 a_{i j} x_{i} x_{j}+a_{j j} x_{j}^{2}$ is reduced we have

$$
-a_{i i}<2 a_{i j} \leqq a_{i i} \quad a_{j j} \geqq a_{i i}
$$

and also

$$
\left|\begin{array}{ll}
a_{\imath i} & a_{i j} \\
a_{i j} & a_{j j}
\end{array}\right|=\left|\begin{array}{cc}
1 & \frac{1}{2} \\
\frac{1}{2} & 1
\end{array}\right|=\frac{3}{4} .
$$

Also it is known that a perfect form is a multiple of an integral form and the minimum of (2.1) is unity, $2 a_{i j}$ is an integer, say $b$.

Thus the above relation gives rise to the Diophantine equation of the type (where $a_{i i}, a_{j j}$ and $b$ are integers)

$$
4 a_{i i} a_{j \jmath}-b^{2}=3
$$

or

$$
4 a_{i i} a_{j j}=3+b^{2} .
$$

This shows that $4 \mid 3+b^{2}$; therefore $b$ is an odd integer, say $2 m+1$, where $m$ is integer.

$$
\begin{aligned}
4 a_{i i} a_{\jmath j} & =3+4 m^{2}+4 m+1 \\
a_{i i} a_{j j} & =m^{2}+m+1 .
\end{aligned}
$$

Also form (3.1) we have

$$
\begin{gathered}
a_{i i} a_{j j} \geqq b^{2}=4 m^{2}+4 m+1 \\
m^{2}+m+1 \geqq 4 m^{2}+4 m+1 \\
3 m(m+1) \leqq 0
\end{gathered}
$$

that is $m=0$ or -1 .

Thus the form (2.1) becomes

$$
x_{i}^{2} \pm x_{i} x_{j}+x_{j}^{2}
$$

The two forms are equivalent and extreme. We now distinguish the various cases. 
4. Case 1.

$$
\begin{array}{ll}
a_{i j}=1 & \text { if } i=j \\
a_{i j}=-\frac{1}{2} & \text { if } i \neq j
\end{array}
$$

In this case

$$
\begin{array}{r}
f_{n}\left(x_{1}, x_{2} \cdots x_{n}\right) \\
=x_{1}^{2}-x_{1} x_{2}-x_{1} x_{3}-\cdots-x_{1} x_{n} \\
+x_{2}^{2}-x_{2} x_{3} \cdots-x_{2} x_{n} \\
+x_{3}^{2}-\cdots-x_{3} x_{n} \\
\cdots \\
\cdots+x_{n}^{2} .
\end{array}
$$

The determinant of (4.1) is

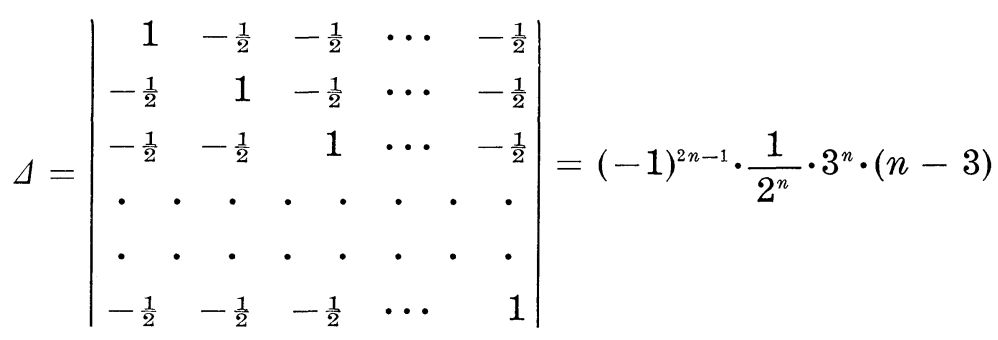

From (4.2) it is clear that (4.1) does not serve our requirement in general.

5. Case 2.

$$
\begin{array}{ll}
a_{i j}=1 & \text { if } i=j \\
a_{i j}=1 / 2 & \text { if } i \neq j
\end{array}
$$

In this case the form is

$$
\begin{array}{r}
f_{n}\left(x_{1}, x_{2} \cdots x_{n}\right) \\
=x_{1}^{2}+x_{1} x_{2}+x_{1} x_{3}+\cdots+x_{1} x_{n} \\
+x_{2}^{2}+x_{2} x_{3}+\cdots+x_{2} x_{n} \\
+x_{3}^{2}+\cdots+x_{3} x_{n} \\
\cdots+x_{n}^{2} .
\end{array}
$$

The determinant of (5.1) is

$$
\Delta=\left|\begin{array}{ccccc}
1 & \frac{1}{2} & \frac{1}{2} & \cdots & \frac{1}{2} \\
\frac{1}{2} & 1 & \frac{1}{2} & \cdots & \frac{1}{2} \\
\frac{1}{2} & \frac{1}{2} & 1 & \cdots & \frac{1}{2} \\
\cdot & \cdot & \cdot & \cdot & \cdot \\
\frac{1}{2} & \frac{1}{2} & \frac{1}{2} & \cdots & 1
\end{array}\right|=\frac{1}{2^{n}}(n+1) .
$$


Clearly we have the following $S=\frac{1}{2} n(n+1)$ representations

$$
\begin{array}{lrl}
x_{i}=1 & x_{j}=0 & i \neq j \\
\left(x_{i}, x_{j}\right)=(1,-1) & i \neq j
\end{array}
$$

and the rest zero.

We notice that

$$
\phi_{n}=x_{1}^{2}-x_{1} x_{2}+x_{2}^{2}-x_{2} x_{3}+\cdots+x_{n}^{2}
$$

has determinant $1 / 2^{n} \cdot(n+1)$ and is equivalent to $(5.1)$. In this case the minimal vectors are:

$$
(1,0,0,0, \cdots, 0)_{n},(1,1,0,0, \cdots, 0)_{n-1}, \cdots,(1,1,1, \cdots, 1,0)_{2}
$$

and $(1,1,1, \cdots, 1)_{1}$, where $\left(x_{1}, x_{2}, \cdots, x_{n}\right)_{t}$ represents the minimal vectors obtained by the cyclic permutation of the variables $0,1,2,3, \cdots$, $t-1$ times. Let $u \dot{\phi}_{n}=\dot{\phi}_{n-1}-x_{t} x_{n}+\frac{1}{2} t\left(1-u^{-1}\right) x_{n}^{2}$

$$
(n=t u-1>1, u>1) \text {. }
$$

It is known that the reciprocal of (5.3) is ${ }^{n+1} \phi_{n}$ and ${ }^{n+1} \phi_{n}$ can be expressed in the form (1.11) and hence (5.1) is extreme, and serves our purpose.

In this connection, it is interesting to note that (5.1) does serve our purpose but its equivalent (5.3) does not serve the requirement of $\S 2$ as can be seen by $f_{2}\left(x_{1}, x_{3}\right)=x_{1}^{2}+x_{3}^{2}$ which is disjoint, hence not perfect.

6. Case 3.

$$
\begin{array}{ll}
a_{i j}=1 & i=j \\
a_{i j}=1 / 2 \text { or }-1 / 2 & i \neq j
\end{array}
$$

(in arbitrary manner).

Lemma. Let

$$
\Delta_{1}=a-x, \Delta_{2}=\left|\begin{array}{cc}
a-x & h \\
h & a-x
\end{array}\right|, \Delta_{3}=\left|\begin{array}{ccc}
a-x & h & g \\
h & b-x & f \\
g & f & c-x
\end{array}\right|,
$$

$$
\Delta_{4}=\left|\begin{array}{cccc}
a-x & h & g & l \\
h & b-x & f & m \\
g & f & c-x & n \\
l & m & n & d-x
\end{array}\right| \text { and so on. }
$$

Then the roots of

$$
\Delta_{1}=0, \Delta_{2}=0, \Delta_{3}=0, \Delta_{4}=0 \text { and so on }
$$


are all real and the roots of any one of them are separated by those of the preceding equation.

The set (6.2) coincides with those of the characteristic equations of quadratic form for various values of $n$. The lemma tells that all the roots are real, but not necessarily positive in this case when $2 a_{i j}=+1$ or -1 arbitrarily. Further from the lemma it follows that if $\Delta_{t}=0$ has a zero root or a negative root then none of the equations

$$
\Delta_{t+1}=0, \Delta_{t+2}=0, \Delta_{t+3}=0
$$

and so on

has all roots positive. We therefore first ascertain the condition under which $\Delta_{t}=0$ has all roots positive and then examine the possibility that the equation $\Delta_{t+1}=0$ has all roots positive. In this connection we note that if in $\Delta_{r}$ we put $x=0$ and $\Delta_{r}$ is negative then the corresponding quadratic form is not positive-definite and in this case the roots of $\Delta_{r}=0$ are not all positive.

For $n=2$ we have only $x^{2} \pm x y+y^{2}$ which are equivalent and the determinant of the form is

$$
D_{2}=\left|\begin{array}{rr}
1 & +\frac{1}{2} \\
+\frac{1}{2} & 1
\end{array}\right| \text {. }
$$

For $n=3$ we consider the determinant

$$
D_{3}=\left|\begin{array}{lll}
1 & \frac{1}{2} & a_{13} \\
\frac{1}{2} & 1 & a_{23} \\
a_{13} & a_{23} & 1
\end{array}\right|=\frac{1}{2^{3}}\left|\begin{array}{lll}
2 & 1 & 2 a_{13} \\
1 & 2 & 2 a_{23} \\
2 a_{13} & 2 a_{23} & 2
\end{array}\right| .
$$

We put $2 a_{13}=t_{1} ; 2 a_{23}=t_{2}$ where the numerical value of $t_{i}(i=1,2)$ is unity.

$D_{3}=\left(1 / 2^{4}\right)\left\{9-\left(2 t_{2}-t_{1}\right)^{2}\right\}$. The permissible values of $t_{1}$ and $t_{2}$ which keep $D_{3}$ nonzero and positive are

$$
\left.\left.\begin{array}{l}
t_{1}=1 \\
t_{2}=1
\end{array}\right\} \quad \text { or } \quad \begin{array}{l}
-1 \\
-1
\end{array}\right\} .
$$

The corresponding positive definite ternary forms are equivalent. We may have then

$$
D_{3}=\left|\begin{array}{ccc}
1 & \frac{1}{2} & \frac{1}{2} \\
\frac{1}{2} & 1 & \frac{1}{2} \\
\frac{1}{2} & \frac{1}{2} & 1
\end{array}\right|
$$

Similarly 


$$
D_{4}=\frac{1}{2^{10}} \cdot \frac{1}{3^{2}} \cdot 2 \cdot 3 \cdot 2^{3}\left[12\left\{2\left(t_{1} t_{2}+t_{2} t_{3}+t_{3} t_{1}\right)-1\right\}\right]
$$

Where, as before, $2 a_{i 4}=t_{i} i=1,2$, and 3 and the numerical value of $t_{i}$ is unity. The permissible values of $t_{1}, t_{2}, t_{3}$ which keep $D_{4}$ positive, are obtained by $t_{1} t_{2}+t_{2} t_{3}+t_{3} t_{1}=3$. We get again two quaternary positive definite forms which are equivalent. Proceeding in this way we have

$$
T_{n}=\left|\begin{array}{ccccc}
2 & 1 & 1 & \cdots & 1 \\
1 & 2 & 1 & \cdots & 1 \\
1 & 1 & 2 & \cdots & 1 \\
\cdot & \cdot & \cdot & \cdot & \cdot \\
\cdot & \cdot & \cdot & \cdot & \cdot \\
1 & 1 & 1 & \cdots & 2
\end{array}\right|=(n+1) \quad\left(T_{n}=2^{n} D_{n}\right)
$$

And we investigate

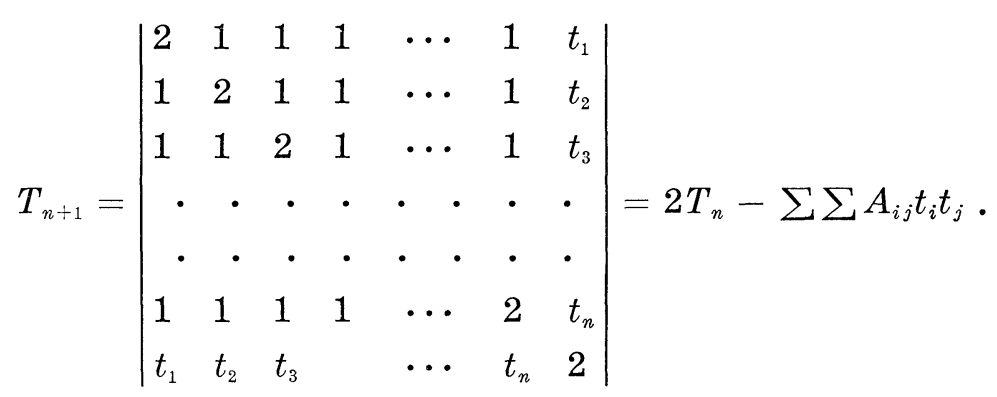

$A_{i j}$ is the cofactor of $a_{i j}$ in $\left|a_{i j}\right|$ where $T_{n} \equiv\left|a_{i j}\right|$. From easy calculation it follows that, in this case $A_{i j}=n$ for every $i$ and $A_{i j}=-1$ for $i=j$

$$
\begin{aligned}
T_{n+1} & =2 T_{n}-\left\{n\left(t_{1}^{2}+t_{2}^{2}+t_{3}^{2}+\cdots+t_{n}^{2}\right)-2\left(t_{1} t_{2} \cdots+t_{1} t_{n}+t_{2} t_{3}+\cdots\right)\right\} \\
& =2(n+1)-\left\{n^{2}-2\left(t_{1} t_{2}+t_{1} t_{3} \cdots+t_{1} t_{n}+t_{2} t_{3}+\cdots\right)\right\} .
\end{aligned}
$$

We have $n$ quantities $t_{i}$ where the numerical value of each $t_{i}=1$ say $r$ of them are each +1 and the remaining $s$ are each -1 ; so $r+s=n$ $\left(r, s\right.$ are positive integers) then the value of the expression $\left(t_{1} t_{2} \cdots+\right.$ $\left.t_{1} t_{n}+t_{2} t_{3}+\cdots\right)$ is

$$
\begin{aligned}
& \frac{r(r-1)}{2}+\frac{s(s-1)}{2}-r s \\
= & \frac{n^{2}}{2}-\frac{n}{2}-2 r s . \quad[r \text { and } s \text { are not zero simultaneously }]
\end{aligned}
$$

Therefore 


$$
\begin{aligned}
T_{n+1} & =2(n+1)-\left\{n^{2}-\left(n^{2}-n-4 r s\right)\right\} \\
& =4 s^{2}-4 n s+n+2 .
\end{aligned}
$$

This expression is to be positive.

Therefore $s<\alpha$ or $s>\beta$ where $\alpha$ and $\beta$ (where $\alpha<\beta$ ) are the roots of

$$
\begin{gathered}
4 s^{2}-4 n s+(n+2)=0 \\
\beta=\frac{n+\sqrt{n^{2}-n-2}}{2}>n-1 \quad \text { if } n>2
\end{gathered}
$$

in this case $s=n$

$$
\alpha=\frac{n-\sqrt{n^{2}-n-2}}{2}<1 \quad \text { if } n>2
$$

in this case $s=0$

$(s=n)$ and $(s=0)$ give two equivalent forms and

$$
T_{n+1}=\left|\begin{array}{ccccc}
2 & 1 & 1 & \cdots & 1 \\
1 & 2 & 1 & \cdots & 1 \\
\cdot & \cdot & \cdot & \cdot & \cdot \\
1 & 1 & & \cdots & 2
\end{array}\right| .
$$

From the above discussion it follows that the solutions of the problem of this paper are given by the forms

$$
\frac{1}{2}\left\{x_{1}^{2}+x_{2}^{2}+\cdots+x_{n}^{2}+\left(x_{1} \pm x_{2} \pm \cdots \pm x_{n}\right)^{2}\right\}
$$

The forms (6.3) are all equivalent to the form $U_{n}$ of Korkine and Zolotareff.

7. REMARK. In this connection it is worth-while to note that the problem of this paper is capable of the following generalization. Find a positive definite (extreme) form $f_{n}$ such that each $f_{r}\left(x_{i}, x_{j}, \cdots, x_{k}\right)$ is extreme (when the number of variables in $f_{r}\left(x_{i}, x_{r}, \cdots, x_{l}\right)$ is $r$; $r<n)$.

It is clear that the form (5.1) gives one answer in every case. Other forms may also be admissible.

For $r=3$ the problem may be tackled in more or less the same way as it is known that all ternary quadratic extreme forms are equivalent to a single class.

$$
x_{1}^{2}+x_{2}^{2}+x_{3}^{2}-x_{1} x_{2}-x_{2} x_{3} .
$$

When $r=4,5$ and 6 the problem may be tackled with great dif- 
ficulties as we know that

(a) When $r=4$ there are two classes of extreme forms equivalent to

$$
\begin{gathered}
x_{1}^{2}+x_{2}^{2}+x_{3}^{2}+x_{4}^{2}+x_{1} x_{2}+x_{1} x_{3}+x_{1} x_{4}+x_{2} x_{3}+x_{2} x_{4}+x_{3} x_{4} \\
x_{1}^{2}+x_{2}^{2}+x_{3}^{2}+x_{4}^{2} \pm x_{1} x_{4} \pm x_{2} x_{4} \pm x_{3} x_{4} .
\end{gathered}
$$

(b) When $r=5$ there are three extreme forms

$$
\begin{gathered}
x_{1}^{2}+x_{2}^{2}+x_{3}^{2}+x_{4}^{2}+x_{5}^{2}+x_{1} x_{2}+x_{1} x_{3}+x_{1} x_{4}+x_{1} x_{5}+x_{2} x_{3} \\
+x_{2} x_{4}+x_{2} x_{5}+x_{3} x_{4}+x_{3} x_{5}+x_{4} x_{5} \\
x_{1}^{2}+x_{2}^{2}+x_{3}^{2}+x_{4}^{2}+x_{5}^{2}-\frac{1}{2} x_{1} x_{2}-\frac{1}{2} x_{1} x_{3}-\frac{1}{2} x_{1} x_{4}-\frac{1}{2} x_{1} x_{5} \\
+\frac{1}{2} x_{2} x_{3}+\frac{1}{2} x_{2} x_{4}-x_{2} x_{5}+\frac{1}{2} x_{3} x_{4}-x_{3} x_{5}-x_{4} x_{5} \\
x_{1}^{2}+x_{2}^{2}+x_{3}^{2}+x_{4}^{2}+x_{5}^{2}+x_{1} x_{3}+x_{1} x_{4}+x_{1} x_{5}+x_{2} x_{3}+x_{2} x_{4} \\
+x_{2} x_{5}+x_{3} x_{4}+x_{3} x_{5}+x_{4} x_{5} .
\end{gathered}
$$

(c) When $r=6$ Professor Barnes has shown that the following extreme forms exist.

$$
\begin{gathered}
\dot{\phi}_{0}=\sum_{1}^{6} x_{i}^{2}+\sum_{i<j} x_{i} x_{j} \\
\phi_{1}=\phi_{0}-x_{1} x_{2} \\
\phi_{3}=\phi_{0}-\frac{1}{2}\left(x_{1} x_{2}+x_{3} x_{4}+x_{5} x_{6}\right) \quad \text { (Kneser and Barnes). } \\
\phi_{4}=\phi_{0}-\frac{1}{2}\left(x_{1} x_{2}+x_{3} x_{4}+x_{3} x_{5}+x_{3} x_{6}+x_{4} x_{5}+x_{4} x_{6}+x_{5} x_{6}\right)
\end{gathered}
$$

(Coxeter).

$$
\phi_{2}=\phi_{0}-x_{1} x_{2}-x_{1} x_{3}
$$

$$
\dot{\phi}_{6}=\phi_{0}-\frac{1}{2}\left(2 x_{1} x_{2}+x_{1} x_{3}+x_{1} x_{6}+x_{2} x_{5}+x_{4} x_{6}+2 x_{5} x_{6}\right) \quad \text { (Barnes). }
$$

For $r \geqq 7$ the number of extreme forms is not known (for still higher values of $r(r \geqq 11) f_{r}$ is in a genus of more than one class) and even if these are known, the problem becomes very complex as the number of extreme forms increases with $r$.

I wish to thank the referee for his helpful suggestions. 


\section{REFERENCES}

1. Barnard and Child, Higher Algebra.

2. E. S. Barnes, Philosophical Transactions of the Royal Society of London, 1957.

3. L. E. Dickson, History of Number Theory, Vol. III

4. A. Korkine and G. Zolotareff, Math. Annalen XI Band, 1877.

Received February 24, 1967.

UNIVERSITY OF RANCHI

BeHAR, INDIA 


\section{PACIFIC JOURNAL OF MATHEMATICS}

\section{EDITORS}

H. ROYDEN

Stanford University

Stanford, California

\section{J. P. Jans}

University of Washington

Seattle, Washington 98105

\section{J. DUGUNDJI}

Department of Mathematics University of Southern California Los Angeles, California 90007

\section{RICHARD ARENS}

University of California

Los Angeles, California 90024

\section{ASSOCIATE EDITORS}

E. F. BECKENBACH

B. H. NEUManN

F. WOLF

K. YosIDA

\section{SUPPORTING INSTITUTIONS}

UNIVERSITY OF BRITISH COLUMBIA CALIFORNIA INSTITUTE OF TECHNOLOGY UNIVERSITY OF CALIFORNIA MONTANA STATE UNIVERSITY UNIVERSITY OF NEVADA NEW MEXICO STATE UNIVERSITY OREGON STATE UNIVERSITY UNIVERSITY OF OREGON OSAKA UNIVERSITY UNIVERSITY OF SOUTHERN CALIFORNIA
STANFORD UNIVERSITY

UNIVERSITY OF TOKYO UNIVERSITY OF UTAH WASHINGTON STATE UNIVERSITY UNIVERSITY OF WASHINGTON AMERICAN MATHEMATICAL SOCIETY CHEVRON RESEARCH CORPORATION TRW SYSTEMS NAVAL WEAPONS CENTER 


\section{Pacific Journal of Mathematics}

\section{Vol. 25, No. $1 \quad$ September, 1968}

Glen Eugene Bredon, Cosheaves and homology................... 1

Robin Ward Chaney, A chain rule for the transformation of integrals in

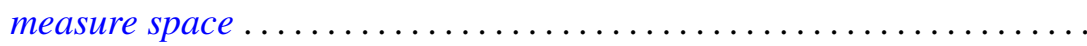

Colin W. Clark, On relatively bounded perturbations of ordinary differential operators................................... 59

John Edwin Diem, A radical for lattice-ordered rings.............. 71

Zeev Ditzian, On a class of convolution transforms ................ 83

Dennis Garoutte and Paul Adrian Nickel, A note on extremal properties characterizing weakly $\lambda$-valent principal functions............. 109

Shwu-Yeng Tzeng Lin, Fixed point properties and inverse limit spaces . . . 117

John S. Lowndes, Some dual series equations involving Laguerre polynomials ................................. 123

Kirti K. Oberai, Sum and product of commuting spectral operators ....... 129

J. N. Pandey and Armen H. Zemanian, Complex inversion for the generalized convolution transformation..................... 147

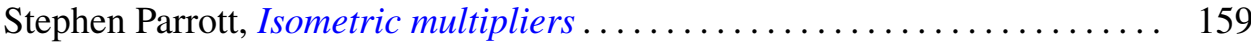

Manoranjan Prasad, Note on an extreme form .................. 167

Maciej Skwarczyński, A representation of a bounded function as infinite product in a domain with Bergman-Shilov boundary surface ......... 177

John C. Taylor, The Šilov boundary for a lattice-ordered semigroup ...... 185 Donald Reginald Traylor and James Newton Younglove, On normality and pointwise paracompactness ............................. 193

L. Tzafriri, Quasi-similarity for spectral operators on Banach spaces ... 